\title{
PARTICULARITIES OF THE SWAMP VEGETATION FROM "IRON GATES" NATURA 2000 SITE, ROMANIA
}

\author{
Irina GOIA ${ }^{1}$, Alexandra ŞUTEU ${ }^{2}$, Miruna GHINDEANU ${ }^{1}$, Adrian OPREA ${ }^{3}$ \\ ${ }^{1}$ Babeș-Bolyai University, Faculty of Biology and Geology, 44, Republicii str., \\ RO-400015 Cluj-Napoca, Romania \\ 2 Babeș-Bolyai University, "Al. Borza" Botanical Garden, \\ 42, Republicii str., RO-400015 Cluj-Napoca, Romania \\ 3 “Alexandru Ioan Cuza” University, “Anastasie Fătu” Botanic Garden, \\ 7-9 Dumbrava Roşie Sreet, RO-700487 Iaşi, Romania \\ e-mail: irina.goia@ubbcluj.ro
}

\begin{abstract}
The swamp vegetation survey was conducted along the Romanian bank of the Danube River, in the Porţile de Fier ("Iron Gates") Natural Park area, in Mehedinţi and Caraş-Severin counties. 20 plant communities and one subassociation belonging to Phragmito-Magnocaricetea, Isoëto-Nanojuncetea and Littorelletea classes were identified. The surveys led to the identification of 8 newly described phytocoenotaxa for this protected area, with one association - Cypero-Paspaletum distichi - being mentioned for the first time in Romania. All the plant communities in this paper are documented by phytosociological tables, being accompanied by coenotaxonomic, phytogeographical and ecological analyses, in order to assess their conservation status, as the main tool for further management decision making.
\end{abstract} allien species

Keywords: Romania, Danube River, "Iron Gates", swamp vegetation, natural habitats, rare species,

\section{Introduction}

The "Iron Gates" Natural Park is one of the most complex systems of rivers, ponds, ditches, and flooded areas, bordered by mountains. Wetlands have covered a larger area since 1972, when one of the largest hydroelectric power plants in Europe, "Iron Gates I", was built on the Danube River. Wetlands are better distributed in the Moldova Veche island and Nera pond, where large areas are flooded during the spring [20].

As a result of the design of the new navigation system in the "Iron Gates", the number of surveys on vegetation has increased, especially in wetlands $[13,21,23,27,29,30,31,33$, $34,24,12]$.

The "Porţile de Fier" Natural Park area has been the subject of many botanical studies. The flora of the "Porţile de Fier" Natural Park consists of 1,875 vascular plant taxa [23], representing more than half of the Romanian flora [32]. Phytosociological surveys encompass 44 plant communities, grouped in two suballiances, 22 alliances, 16 orders, and 13 classes of vegetation [23].

After Romania joined the EU, one of the most important projects regarding the adequate conservation of natural heritage was the development of management plans, which included the conservation status assessment of species and habitats of community interest as a mandatory stage [20].

The objectives of the current study are the reassessment of previously identified 
swamp plant communities, the identification of new plant communities in the area and the analysis of their structure, primarily to highlight the conservation status of habitats, while emphasizing the presence of invasive plant species.

\section{Material and Methods}

The surveys were mainly conducted in the Natura 2000 site "ROSPA0026 Danube sector - Baziaş-Porţile de Fier", largely overlapping the "Iron Gates" Natural Park. The above mentioned site has an area of 9,904 ha, and is located on the territory of Caraş-Severin $(56 \%)$ and Mehedinţi (44\%) counties (south-western Romania), in the Continental biogeographical region. Elevation ranges from 28 to $192 \mathrm{~m}$ a.s.1. [40, 41].

\section{Physical geographic features of the studied area}

The surveyed territory is traversed by the Danube River over a length of $134 \mathrm{~km}$, generating the most spectacular gorge sector in Europe.

From a geological point of view, the area has a mosaic feature: between Coronini and Liuborajdea, the Danube river passes through a limestone sector; west of Liubcova, the Danube crosses a granite area, and toward Cozla, rapids and transverse currents occur due to the substrate formed by gneisses of Ielova, conglomerates, porphyry and porphyrites; downstream of Cozla, the Danube cuts through another limestone area.

The most spectacular section of the Danube River is represented by the so-called "Cazanele Mari" and "Cazanele Mici" areas. Also, from a geological point of view, there are remarkable suspended synclinals called "Munteana", fossiliferous outcrops at Sviniţa and Bahna, a Permian volcanic neck at Trescovăţ, and a variety of karst formations. In some areas, loess deposits are exposed [5, 38]. Moldova Veche island was intended to become a place for storing the mining waste from the "MoldaMin" Mining Company, which exploited copper in Moldova Nouă and coal in Cozla, Baia Nouă and Bigăr.

The average annual temperatures in the "Iron Gates" Natural Park are: Berzasca $11.4^{\circ} \mathrm{C}$, Orşova $11.6^{\circ} \mathrm{C}$, Sviniţa $11.5^{\circ} \mathrm{C}$ (for the period $1970-2000$ ). The average annual rainfall is $586.3 \mathrm{~mm}$ in Orşova and $652.4 \mathrm{~mm}$ in Drobeta-Turnu Severin. The prevailing winds are from north-west in Orşova, and from west and north-west in Drobeta-Turnu Severin [23].

The most widespread zonal soils are clay-illuvial podzols, brown-acid soils, and intrazonal lithomorphic soils. Flooded areas are dominated by alluvial soils, which vary in texture and evolution: sandy silt layered alluvia, alluvial humiferous soils, and brown alluvial soils. On alluvial cones and tributary floodplains, soils are coarse, and on calcareous gravel, the so-called branciogs are found. Alluvial gleyic and even swamp gleyic soils are reported in some places $[23,19]$.

\section{The methodology of the vegetation survey}

83 relevés were sampled according to Braun-Blanquet's method [4]. Identification of plant communities was based on the diagnostic species, also taking into account the presence of the dominant species. In order to draw up the coenotaxonomic conspectus, various records $[8,9,10,25]$, as well as other current available European classifications were consulted.

The area of the samples was related to the size of the phytocoenoses, ranging from 
$1 \mathrm{~m}^{2}$ to $25 \mathrm{~m}^{2}$.

Habitat identification was based on the characteristic phytocoenotaxa (associations, alliances, orders), as stated by the Romanian interpretation manual of habitats $[18,15,16]$, in accordance with the European Union Habitat Directive 92/43/EEC [39].

The vascular flora nomenclature is in accordance with Flora Europaea [35, 42], and with some Romanian identification field books [7, 32]. We used the nomenclature of algae suggested by Cărăuş (2012). The values of ecological and floristic element indexes were established using Popescu and Sanda (1998).

Coenotaxonomic classification was performed using the PAST 3.17 program [22], with the (constrained) UPGMA algorithm and Bray-Curtis quantitative index.

\section{Results}

20 swamp associations and one subassociation distinctively grouped in cluster analyses (Fig. 7) were identified in the area, all of them being assigned to three classes, five orders and eight alliances, according to the following coenotaxonomic conspectus:

${ }^{*}$ newly recorded plant communities in the studied area

\section{PHRAGMITO-MAGNOCARICETEA Klika in Klika et Novák 1941}

Phragmitetalia Koch 1926

Phragmition communis Koch 1926

1. Scirpo-Phragmitetum W. Koch 1926

2. Schoenoplectetum lacustris Chouard 1924

3. Sparganietum erecti Roll 1938

4. Typhetum angustifoliae Pign. 1953

5. Typhetum latifoliae Nowiński 1930

6. ${ }^{*}$ Typhetum laxmannii Nedelcu 1969

7. Glycerietum maximae Nowiński 1930 corr. Šumberová, Chytrý et Danihelka in Chytrý 2011

8. ' Iretum pseudacori Eggler 1933

9. Acoretum calami Schulz 1941

10. * Cyperetum (Juncelletum) serotini Krausch 1965

Meliloto dentati-Bolboschoenion maritimi Hroudová et al. 2009

11. Bolboschoenetum maritimi Eggler 1933

12. *Bolboschoenetum maritimi Eggler 1933 subass. schoenoplectetosum triquetri Coldea et al. 1997

13. * Schoenoplectetum tabernaemontani Soó 1947

Nasturtio-Glycerietalia Pignatti 1953

Glycerio-Sparganion Br.-Bl. et Sissing in Boer 1942

14. Leersietum oryzoidis Eggler 1933

Magnocaricetalia Pignatti 1953

Magnocaricion elatae Koch 1926

15. Caricetum gracilis (Almquist 1929) Tx. 1937

16. Caricetum ripariae Knapp et Stoffer 1962

17. Caricetum vulpinae Nowinski 1927

Eleocharito palustris-Sagittarion sagittifoliae Passarge 1964

18. Eleocharitetum palustris Savič 1926 
ISOËTO-NANOJUNCETEA Br.-Bl. et Tx. in Br.-Bl. et al. 1952

Nanocyperetalia Klika 1935

Nanocyperion Koch 1926

19. * Cyperetum flavescentis W. Koch ex Aichinger 1933

Verbenion supinae Slavnić 1951

20. ${ }^{*}$ Cypero-Paspaletum distichi Horv. 1954

LITTORELLETEA UNIFLORAE Br.-Bl. et Tx. ex Westhoff et al. 1946

Littorelletalia Koch ex Tx. 1937

Eleocharition acicularis Pietsch 1967

21. * Eleocharitetum acicularis Koch 1926 em. Oberd. 1957

Class Phragmito-Magnocaricetea Klika in Klika et Novák 1941. Plant communities dominated by reedbeds and sedges are widespread in the studied area. This class is represented by three orders: Phragmitetalia, Nasturtio-Glycerietalia, and Magnocaricetalia. Of all 149 species, only 35 are characteristic of the class or coenotaxonomic unit components, which indicates permanent pressures, either anthropogenic or natural, due to interference with other communities (Tables 1, 2). Cluster analyses highlight this heterogeneity (Fig. 7). Most of the identified phytocoenotaxa of this class are found on the Moldova Veche island, in the Nera delta and Divici pond.

Order Phragmitetalia Koch 1926 (Table 1) is represented in the "Iron Gates" Natural Park by 2 alliances (Phragmition and Meliloto dentati-Bolboschoenion maritimi). Alliance Phragmition Koch 1926 includes generally monodominant communities, composed of tall graminoid species. This alliance is the best grouped in cluster analyses (Fig. 7). Alliance Meliloto dentati-Bolboschoenion maritimi Hroudová et al. 2009 includes communities that show a moderate to rich salt content, with seasonal fluctuations of water levels; most often water dries up at the surface, but still persists in the soil.

Order Nasturtio-Glycerietalia Pignatti 1953 (Table 2) includes plant communities that are flooded only in the spring. The only alliance Glycerio-Sparganion Br.-Bl. et Sissing in Boer 1942 includes plant communities settled in small depression areas.

Order Magnocaricetalia Pignatti 1953 (Table 2) includes plant communities settled on moist, humus-rich alluvial soils, occasionally flooded or with a permanent shallow water layer. In terms of succession, these communities have established after Phragmitetalia communities. Alliance Magnocaricion Koch 1926 includes plant communities developed on neutral or slightly acidic soils.

Eurasian species (especially those with a Mediterranean characteristic) are very well represented, mainly in phytocoenoses of the Phragmitetalia order (Fig. 1). The \pm homogeneous features of the swamps are highlighted by a good representation of cosmopolitan species. Circumpolar species find a refuge in the shade of the large species of plant communities in this class. Adventive species (13 species) are constantly present in the phytocoenoses of the Phragmitetea class. Among these, Amorpha fruticosa, Ambrosia artemisiifolia, Erigeron strigosus and Paspalum paspalodes are definetely invasive, while Erigeron annuus, Bidens vulgata, Galinsoga parviflora, Oenothera parviflora, Echinocystis lobata, Amaranthus retroflexus and Xanthium italicum are potentially invasive. Sagittaria trifoliata, Acorus calamus and Azolla filiculoides are poorly represented in swamp phytocoenoses. 


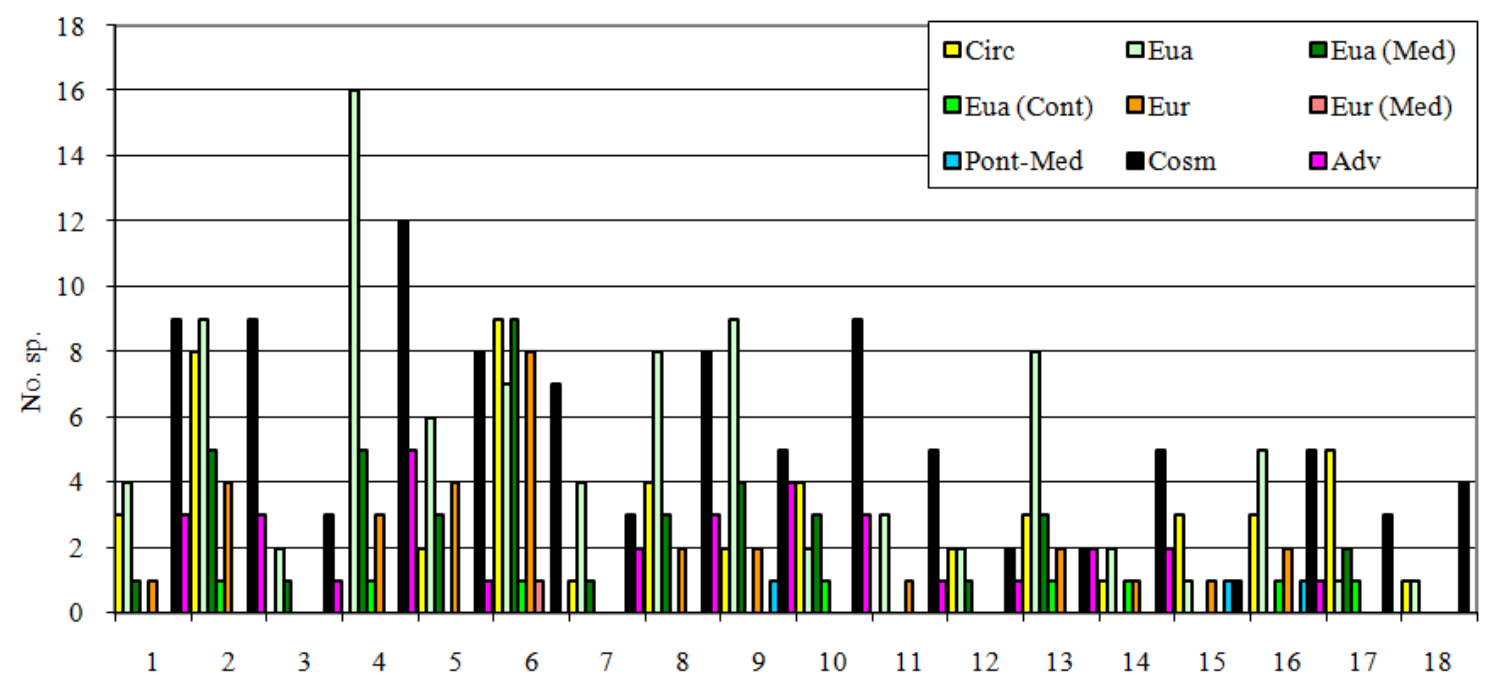

Fig. 1: The phytogeographical spectrum of Phragmito-Magnocaricetea communities from the "Iron Gates" Natural Park. 1. Scirpo-Phragmitetum 2. Schoenoplectetum lacustris 3. Sparganietum erecti 4. Typhetum angustifoliae 5. Typhetum latifoliae 6. Typhetum laxmanii 7. Glycerietum maximae 8. Iridetum pseudachori 9. Acoretum calami 10. Cyperetum serotini 11. Bolboschoenetum maritimi 12. Bolboschoenetum maritimi schoenoplectetosum triquetri 13. Schoenoplectetum tabernaemontani 14. Leersietum oryzoidis 15. Caricetum gracilis 16. Caricetum ripariae 17. Caricetum vulpinae 18. Eleocharitetum palustris.

The ecological spectrum (Fig. 2) indicates hydrophilous $\left(\mathrm{U}_{6}\right)$ to hygrophilous $\left(\mathrm{U}_{5-5.5}\right)$ characteristics of phytocoenoses in the Phragmitetea class (Fig. 3). The fluctuating level of waters is reflected by the presence of mesophilic $\left(\mathrm{U}_{3-3.5}\right)$ or xero-mesophilic $\left(\mathrm{U}_{2-2.5}\right)$ species, or even by the predominance of meso-hygrophilous species (in Schoenoplectetum tabernaemontani, where soil salt content may be responsible for this situation).

The temperate climate of the "Iron Gates" area is reflected by the predominance of micro-mesothermal species $\left(\mathrm{T}_{3-3.5}\right)$. The influence of the Mediterranean climate is evidenced by the presence of moderately thermophilic $\left(\mathrm{T}_{4-4.5}\right)$ and thermophilic $\left(\mathrm{T}_{5}\right)$ species (Fig. 2). Large masses of the dominant species allow the shading and settlement of some microthermal species $\left(\mathrm{T}_{2-2.5}\right)$.

Relative to the soil $\mathrm{pH}$ (Fig. 2), euriionic species $\left(\mathrm{R}_{0}\right)$ are predominant, being followed by weakly acid to neutrophilic species $\left(\mathrm{R}_{4-4.5}\right)$. Of stenoionic species, in many communities, acid neutrophilic species $\left(\mathrm{R}_{3-3.5}\right)$ are well-represented, followed by neutro-basophilic species

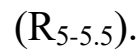

Class Isoëto-Nanojuncetea Br.-Bl. et Tx. in Br.-Bl. et al. 1952. This class includes small plant communities, mostly graminoid species and dicotyledonous therophytes, growing in small moist depressions, as well as on fine sludge from former lakes or river banks under regular floods. These communities are poorly represented in the studied area, displaying a better occurence on the Moldova Veche island (Table 3). These are pioneering communities, evolving depending on the influence of edaphic and hydric factors to phytocoenoses belonging to different classes. Only 9 of the 75 species are characteristic of the class or coenotaxonomic unit components, as an argument of the fast succession of these communities related to the water table. Better represented are diagnostic species of MolinioArrhenatheretea and Bidentetea classes. 
Table 1: Plant communities of All. Phragmition communis Koch 1926 (part 1)

\begin{tabular}{|c|c|c|c|c|c|c|c|c|c|c|c|c|c|c|c|c|c|c|c|c|c|c|c|c|c|c|c|c|c|c|}
\hline Relevé no. & 1 & 2 & 3 & 4 & 5 & 6 & 7 & 8 & 9 & 10 & 11 & 12 & 13 & 14 & 15 & 16 & 17 & 18 & 19 & 20 & 21 & 22 & 23 & 24 & 25 & 26 & 27 & 28 & 29 & 30 \\
\hline Relevé area $\left(\mathrm{m}^{2}\right)$ & 25 & 25 & 25 & 9 & 9 & 6 & 25 & 25 & 25 & 9 & 9 & 9 & 25 & 25 & 25 & 9 & 25 & 9 & 9 & 9 & 9 & 25 & 9 & 25 & 25 & 25 & 25 & 25 & 25 & 25 \\
\hline \begin{tabular}{|l|} 
Total cover $(\%)$ \\
\end{tabular} & 100 & 100 & 80 & 70 & 90 & 70 & 80 & 100 & 100 & 80 & 95 & 100 & 100 & 50 & 100 & 100 & 95 & 80 & 85 & 100 & 100 & 100 & 90 & 90 & 85 & 90 & 90 & 90 & 100 & 95 \\
\hline Phragmites australis & 5 & 5 & 4 & 3 & & + & $\cdot$ & $\cdot$ & $\cdot$ &. &. & . & . & & . & . & 1 & . & . & . & $\cdot$ & . & $\cdot$ & . & . & $\cdot$ & . & & + & . \\
\hline Schoenoplectus lacustris & . & . & . & . & 4 & 4 & 5 & 3 & 3 & + & . & 1 & . & . & . & . & . & . & . & . & . & . & . & . & . & . & . & . & . & . \\
\hline Typha angustifolia & . & . & 2 & . & 1 & . & + & . & . & . & 5 & 5 & 5 & 3 & 5 & 5 & 4 & 5 & + & . & . & . & . & . & . & . & . & . & . & . \\
\hline Typha latifolia & . & . & . & 1 & 2 & 1 & . & . & . & . & . & . & . & . & . & . & . & . & 4 & 5 & 5 & 5 & 4 & . & . & . & . & & . & . \\
\hline Typha laxmannii & . & . & . & . & . & . & . & + & . & . & . & + & . & . & . & . & . & . & . & . & . & . & . & 4 & 4 & 4 & 4 & 5 & 5 & 5 \\
\hline \multicolumn{31}{|l|}{ Pragmition } \\
\hline Alisma plantago-aquatica & . & . & . & . & . & . & . & + & 1 & . & . & . & + & 1 & . & . & . & . & + & . & . & . & . & . & . & . & 1 & + & + & . \\
\hline Butomus umbellatus & . & . & + & . & . & + & + & . & . & + & + & + & . & . & . & + & . & + & . & . & . & . & . & . & . & . & . & + & . & . \\
\hline Oenanthe aquatica & . & . & . & . & . & . & + & . & . & . & . & . & . & . & . & . & . & . & . & . & . & . & . & . & . & . & . & . & . & . \\
\hline Rumex hydrolapathum & . & . & . & . & . & . & + & . & . & . & . & . & + & + & . & . & . & + & . & . & . & . & . & . & . & . & . & . & . & . \\
\hline Sium latifolium & . & . & . & . & . & . & + & . & . & . & . & . & . & . & . & . & + & + & . & . & . & . & . & . & . & . & . & . & . & . \\
\hline Solanum dulcamara & . & . & . & . & . & . & . & . & . & . & . & . & . & . & + & . & . & . & + & . & . & + & . & . & . & . & . & . & . & . \\
\hline Sparganium erectum & . & . & . & . & . & . & . & . & . & 3 & . & . & . & . & . & . & . & + & . & . & . & + & . & . & . & . & . & . & . & . \\
\hline \multicolumn{31}{|c|}{ Meliloto dentati-Bolboschoenion maritimi } \\
\hline Carex otrubae & . & . & . & . & . & . & + & . & . & . & . & . & . & + & . & . & . & + & . & . & . & . & . & + & 1 & + & . & . & . & . \\
\hline $\begin{array}{l}\text { Bolboschoenus maritimus } \\
\text { Bolboschoenus maritimus f. }\end{array}$ & . & . & . & . & . & . & . & . & . & . & . & . & . & . & . & . & + & . & . & . & . & . & + & . & . & . & . & . & . & . \\
\hline macrostachys & . & . & . & + & . & . & . & . & . & . & . & . & . & . & . & . & . & . & . & . & . & . & . & $\cdot$ & . & . & . & . & . & . \\
\hline Juncus gerardii & . & . & . & . & . & . & . & . & . & . & . & . & . & . & . & . & . & . & . & . & . & . & . & + & . & . & . & . & . & . \\
\hline Schoenoplectus tabernaemontani & . & . & . & . & . & . & . & . & . & . & . & . & . & . & . & . & . & . & . & . & . & . & . & . & . & . & . & . & + & . \\
\hline Schoenoplectus triqueter & . & . & . & . & . & . & . & . & . & . & . & . & . & + & . & . & . & . & . & . & . & . & . & . & . & . & . & . & . & . \\
\hline \multicolumn{31}{|l|}{ Magnocaricion } \\
\hline Cicuta virosa & . & . & . & . & . & . & . & . & . & . & + & . & . & . & . & . & . & . & . & . & . & 1 & . & . & . & . & . & . & . & . \\
\hline Cirsium palustre & . & . & . & . & . & . & . & . & . & . & . & . & . & . & . & . & . & . & . & . & . & . & . & . & . & . & + & . & . & . \\
\hline Galium palustre & . & . & + & . & . & + & . & . & . & . & . & . & . & + & . & + & . & . & . & . & . & . & . & + & + & + & + & + & . & . \\
\hline Poa palustris & . & . & . & . & . & . & . & . & . & . & + & . & . & . & . & . & . & . & . & . & . & . & . & . & . & . & . & . & . & . \\
\hline Scutellaria galericulata & . & . & . & . & . & . & . & . & + & . & . & . & + & $\cdot$ & . & . & . & . & . & . & . & . & . & . & . & . & + & + & . & . \\
\hline \multicolumn{31}{|c|}{ Phragmitetalia et Phragmito-Magnocaricetea } \\
\hline Glyceria maxima & . & . & + & . & . & + & . & $\cdot$ & . & . & . & . & . & . & . & . & + & . & . & . & . & . & . & . & . & . & . & . & . & $\cdot$ \\
\hline Iris pseudacorus & . & . & . & . & . & . & . & 2 & 1 & . & . & . & . & . & . & . & . & . & + & . & . & . & $\cdot$ & + & . & . & . & . & . & + \\
\hline Lycopus europaeus & + & + & . & . & . & . & . & + & + & . & + & + & + & + & + & . & + & . & 1 & . & . & . & + & 1 & . & + & 1 & + & . & + \\
\hline Mentha aquatica & . & . & . & + & . & . & . & 1 & 2 & . & + & + & . & $\cdot$ & + & + & . & . & + & . & . & + & & + & . & + & . & & & . \\
\hline
\end{tabular}


Phalaris arundinacea

Rorippa amphibia

Stachys palustris

Veronica anagallis-aquatica

Bidention et Bidentetea

Bidens cernua

Bidens tripartita

Chenopodium album

Polygonum hydropiper

Polygonum persicaria

Rorippa sylvestris

Rumex palustris

Sonchus asper

Xanthium italicum

Nanocyperion et Nanocyperetalia

Cyperus flavescens

Cyperus fuscus

Cyperus serotinus

Mentha pulegium

Pulicaria vulgaris

Litorelletea

Eleocharis acicularis

Marsilea quadrifolia

Lemnetalia et Lemnetea

Lemna minor

Salvinia natans

Spirodela polyrhiza

Utricularia vulgaris

Molinio-Arrhenathetea

Agrostis stolonifera

Alopecurus geniculatus

Calystegia sepium

Carex hirta

Epilobium hirsutum

Epilobium parviflorum

Equisetum palustre

Gratiola officinalis

Juncus articulatus

Juncus effusus 
Juncus inflexus

Lysimachia nummularia

Lythrum salicaria

Mentha longifolia

Myosotis scorpioides

Potentilla reptans

Pulicaria dysenterica

Scirpus sylvaticus

Salicetea purpureae

Salix purpurea

Aliae

Amorpha fruticosa

Calamagrostis epigejos

Drepanocladus aduncus

Echinocystis lobata

Equisetum arvense

Equisetum ramosissimum

Eupatorium cannabinum

Galinsoga parviflora

Mentha verticillata

Paspalum paspalodes

Salix alba

Scirpus carinatus

Trapa natans

Species with one occurrence: Alopecurus geniculatus $+(12)$; Bidens vulgata $+(12)$; Chaenarrhinum minus $+(26) ;$ Cirsium arvense $+(26)$; Cladophora glomerata $+(21)$; Cruciata glabra + (30); Epilobium palustre + (27); Erigeron annuus $+(12)$; Lysimachia vulgaris $+(13) ;$ Juncus bulbosus $+(24) ;$ Leersia oryzoides $+(11) ;$ Potamogeton pectinatus $+(20) ;$ Rubus hirtus $+(27) ;$ Scirpus holoschoenus $+(24)$.

Date and place of relevés: Ass.1. Scirpo-Phragmitetum: rel. 1- Belobreșca pound, rel. 2 - Divici pound, rel. 3-4 - Nera pound, (27.06.2012); Ass. 2. Schoenoplectetum lacustris: rel. 5- Pojejena pound (25.05.2012), rel. 6 - Divici pound (27.06.2012), rel. 7- Nera pound (27.06.2012), rel. 8-9 - Moldova Veche island (31.07.2012); Ass. 3. Sparganietum erecti: rel. 10 - Nera pound

(27.06.2012); Ass. 4. Typhetum angustifoliae: rel. 11 - Cerna Golf (23.06.2012), rel. 12 - Liubcova (27.06.2012), rel 13-14 - Moldova Veche island (25.06.2012), rel. 15 - Divici pound

(27.06.2012), rel. 16-18 - Nera pound (27.06.2012); Ass. 5. Typhetum latifoliae: rel. 19 - Ponicova (24.06.2012), rel. 20 - Belobresca pound (25.06.2012), rel. 21- Calinovăț Holm (25.06.2012),

rel. 22-23 - Divici pound (27.06.2012); Ass. 6. Typhetum laxmannii - Moldova Veche island: rel. 24-26 (26.06.2012), rel. 27-28 (31.07.2012), rel. 29-30 (23.09.2012). 
Table 2: Plant communities of All. Phragmition communis Koch 1926 (part 2), All. Meliloto dentati-Bolboschoenion maritimi Hroudová et al. 2009, All. Glycerio-Sparganion Br.-Bl. et Sissing in Boer 1942, All. Magnocaricion elatae Koch 1926

Relevé no.

$\begin{array}{lllllllllllllllllllllllllll}31 & 32 & 33 & 34 & 35 & 36 & 37 & 38 & 39 & 40 & 41 & 42 & 43 & 44 & 45 & 46 & 47 & 48 & 49 & 50 & 51 & 52 & 53 & 54 & 55 & 56\end{array}$

Relevé area $\left(\mathrm{m}^{2}\right)$

$\begin{array}{lllllllllllllllllllllllllll}25 & 25 & 9 & 25 & 25 & 25 & 15 & 25 & 25 & 25 & 9 & 9 & 25 & 9 & 9 & 9 & 25 & 25 & 25 & 9 & 25 & 25 & 25 & 25 & 3 & 3\end{array}$

Total cover (\%)

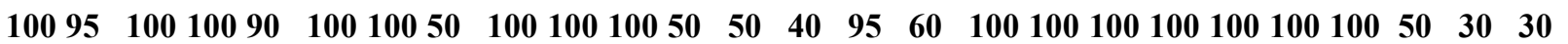

Glyceria maxima

Iris pseudacorus

Acorus calamus

Cyperus serotinus

Bolboschoenus maritimus

Schoenoplectus triqueter

Schoenoplectus tabernaemontani

Leersia oryzoides

Carex acuta

Carex riparia

Carex vulpina

Eleocharis palustris

Phragmition

Alisma gramineum

Alisma plantago-aquatica

Rumex hydrolapathum

Sparganium erectum

Typha angustifolia

Typha latifolia

Butomus umbellatus

Meliloto dentati-Bolboschoenion maritimi

Bolboschoenus maritimus f. macrostachys

Carex otrubae

Juncus gerardii

Magnocaricion

Cicuta virosa

Galium palustre

Poa palustris

Phragmitetalia et Phragmitetea

Lycopus europaeus

Phalaris arundinacea

Phragmites australis

$\begin{array}{llllll}5 & 5 & . & . & . & 1 \\ . & . & 5 & 4 & 3 & 5\end{array}$

$\begin{array}{ccccccc}100 & 50 & 100 & 100 & 100 & 50 & 50 \\ .3 & . & . & . & . & . & . \\ . & . & . & . & . & . & . \\ . & 3 & 3 & 2 & . & . & . \\ . & . & . & . & 4 & 1 & 3 \\ . & . & . & . & . & . & + \\ . & . & . & . & . & + & \\ . & . & . & . & + & 1 & \end{array}$

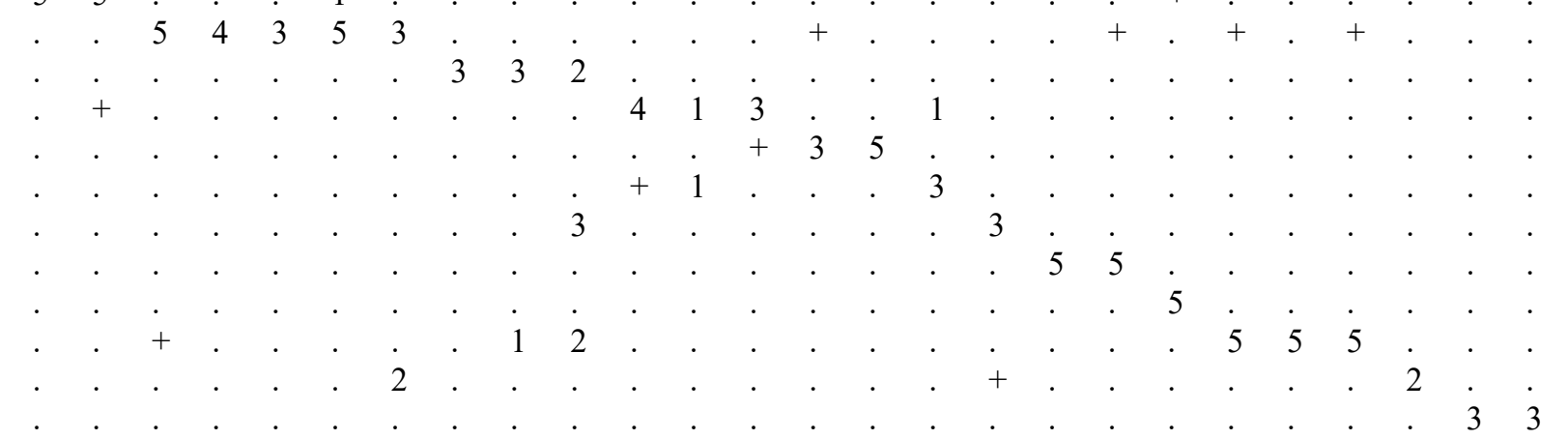


Schoenoplectus lacustris

Mentha aquatica

Stachys palustris

Typha laxmannii

Bidention et Bidentetea

Amaranthus retroflexus

Bidens cernua

Bidens tripartita

Echinochloa crus-galli

Galega officinalis

Polygonum hydropiper

Rorippa sylvestris

Rumex palustris

Molinietalia et Molinio-Arrhenatheretea

Agrostis stolonifera

Althaea officinalis

Calystegia sepium

Carex hirta

Epilobium hirsutum

Equisetum palustre

Gratiola officinalis

Juncus articulatus

Juncus compressus

Juncus effusus

Juncus inflexus

Lysimachia nummularia

Lythrum salicaria

Myosotis scorpioides

Potentilla anserina

Potentilla reptans

Prunella vulgaris

Ranunculus repens

Trifolium hybridum

Nanocyperetalia et Isoëto-Nanojuncetea

Cyperus flavescens

Mentha pulegium

Pulicaria vulgaris

Litorelletea

Eleocharis acicularis

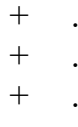


Marsilea quadrifolia

Potametea

Myriophyllum spicatum

Najas marina

Najas minor

Potamogeton nodosus

Potamogeton pectinatus

Potamogeton perfoliatus

Drepanocladus aduncus

Lemnetalia et Lemnetea

Lemna minor

Salvinia natans

Spirodela polyrhiza

Azolla filiculoides

Aliae

Amorpha fruticosa

Chara foetida

Erigeron strigosus

Leptodictyum riparium

Lysimachia vulgaris

Paspalum paspaloides

Salix cinerea

Solanum dulcamara

Urtica dioica

Vitis vinifera

Species with one occurrence: Elymus repens 2 (35); Ambrosia artemisiifolia $+(41)$; Calliergonella cuspidata + (47); Carex distans 2 (54); Eupatorium

cannabinum + (47); Glechoma hederacea + (34); Mentha verticillata 2 (47); Moehringia trinervia + (39); Oenothera parviflora + (40); Plantago major + (40);

Polygonum lapathifolium + (34); Portulaca oleracea + (49); Potentilla argentea + (35); Rumex obtusifolius + (50); Sagittaria trifoliata + (47); Salix alba + (47);

Setaria glauca $+(41) ;$ Sium sisarum $+(48) ;$ Xanthium strumarium $+(38)$.

Date and place of relevés: Ass. 7. Glycerietum maximae: rel. 31 - Divici pound (27.06.2012), rel. 32 - Nera pound (2.06.2012); Ass. 8. Iridetum pseudachori: rel. 33 - Liubotina Valley (24.06.2012), rel. 34 - between Divici and Bazias (27.06.2012), rel. 35-36 - Nera pound (27.06.2012), rel. 37 - Moldova Veche Island (23.09 2012); Ass. 9. Acoretum calami: rel 38-40 - Nera pound (27.06.2012); Ass. 10. Cyperetum serotini: rel. 41- Eselnita (25.06.2012), rel. 42-43 Moldova Veche island (31.07.2012); Ass.11. Bolboschoenetum maritime: rel 44 - Divici pound (27.06.2012), rel. 45 - Nera pound (27.06.2012); Ass.12. Bolboschoenetum maritimi schoenoplectetosum triquetri: rel. 46 - Moldova Veche island (31.07.2012); Ass. 13. Schoenoplectetum tabernaemontani: rel. 47 - Moldova Veche island (31.07.2012); Ass. 14. Leersietum oryzoidis: rel. 48 - Nera pound (22.09.2012), rel. 49 - Pojejena (24.09.2012); Ass. 15. Caricetum gracilis: rel. 50 - Divici pound (27.06.2012); Ass. 16. Caricetum ripariae: rel.51 - Liubotina Valley (25.06.2012), rel. 52 - Nera pound (27.06.2012), rel. 53 - between Divici and Bazias (27.06.2012); Ass. 17. Caricetum vulpinae: rel. 54 - Moldova Veche island (26.06.2012); Ass. 18. Eleocharitetum palustris: rel. 55-56 - Moldova Veche island (26.06.2012). 


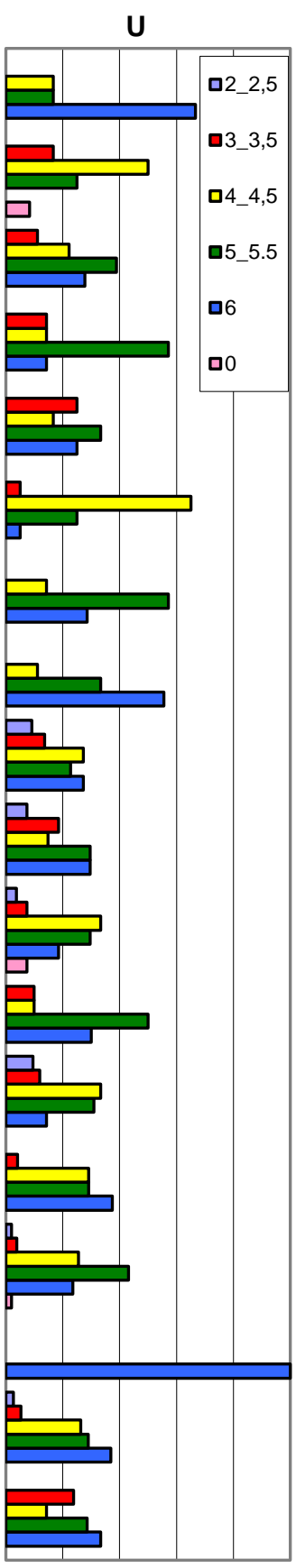

$\%$

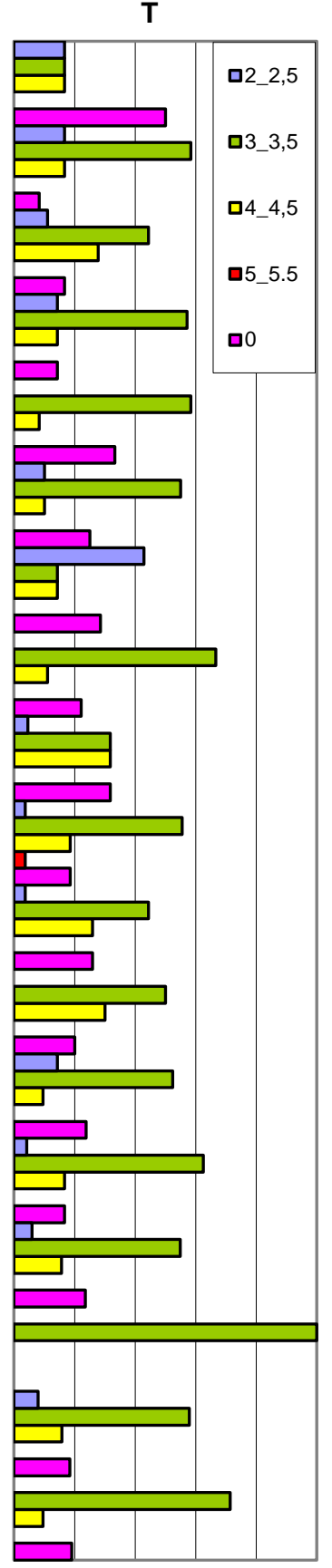

$\%$

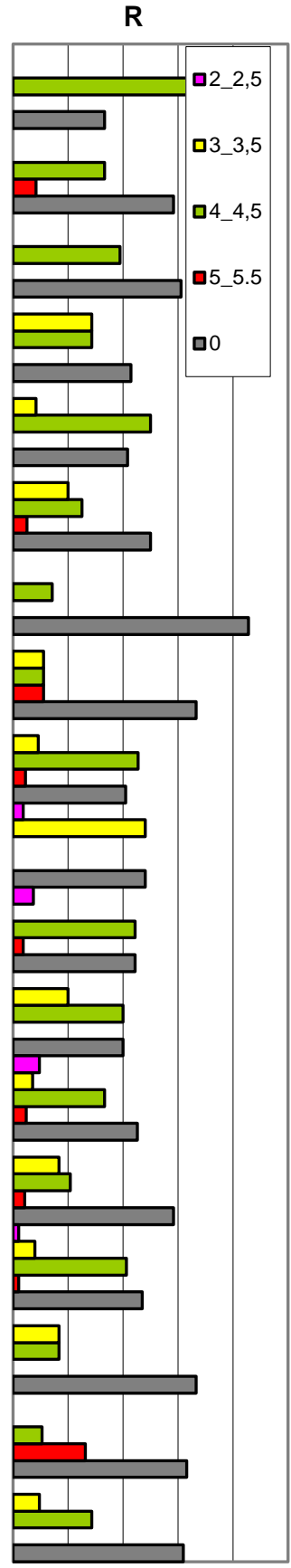

$\%$

Fig. 2: The ecological spectrum of Phragmito-Magnocaricetea communities. 1. Scirpo-Phragmitetum 2. Schoenoplectetum lacustris 3. Sparganietum erecti 4. Typhetum angustifoliae 5. Typhetum latifoliae 6. Typhetum laxmanii 7. Glycerietum maximae 8. Iridetum pseudachori 9. Acoretum calami 10. Cyperetum serotini 11. Bolboschoenetum maritimi 12. Bolboschoenetum maritimi schoenoplectetosum triquetri 13. Schoenoplectetum tabernaemontani 14. Leersietum oryzoidis 15. Caricetum gracilis 16. Caricetum ripariae 17. Caricetum vulpinae 18. Eleocharitetum palustris.

Order Nanocyperetalia Klika 1935 consists of annual Cyperaceae and Juncaceae, settled on flooded, calcium-rich, formerly muddy substrates. Alliance Nanocyperion Koch is the only alliance of this class represented in the "Iron Gates" Natural Park. This alliance is represented by the phytocoenoses of two communities: Cyperetum flavescentis W. Koch ex 
Aisinger 1933 and Cypero-Paspaletum distichi Horv. 1954, the latter being reported for the first time in Romania's vegetation.

The analysis of phytogeographical elements reflects the predominance of cosmopolitan rather than Eurasian elements, with a good representation of Mediterranean elements, especially in Cyperetum flavescentis phytocoenoses (Fig. 3). 13 alien species were identified in these associations; even if some of them (Ambrosia artemisiifolia, Amorpha fruticosa, Erigeron strigosus, and Euphorbia maculata) are known to be invasive, here they are less represented.

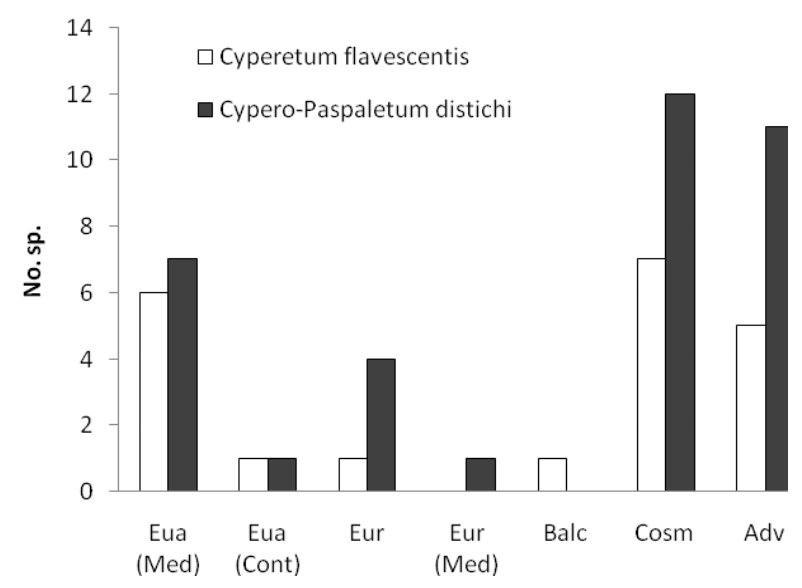

Fig. 3: The phytogeographical spectrum of Isoëto-Nanojuncetea communities from the "Iron Gates" Natural Park

From an ecological point of view (Fig. 4), meso-hygrophilic elements ( $\left.\mathrm{U}_{4-5.5}\right)$ predominate in co-dominance with mesophilic elements $\left(\mathrm{U}_{3-3.5}\right)$ in Cyperetum flavescentis and Cypero-Paspaletum distichi phytocoenoses. The temperature preferences of the species in the communities of this class give it a micro-mesothermal feature $\left(\mathrm{T}_{3-3.5}\right)$, with a good representation of moderately thermophilic species $\left(\mathrm{T}_{4-4.5}\right)$. Regarding the chemical reaction of the soil, euriionic elements $\left(\mathrm{R}_{0}\right)$ are predominant, followed by weakly neutrophilic acidic species $\left(\mathrm{R}_{4-4.5}\right)$.

Class Litorelletea uniflorae Br.-Bl. et Tüxen ex Westhoff et al. 1946 includes plant communities that develop on lake shores, being periodically flooded, on sandy-clayey or peaty soils. Of diagnostic species, only Eleocharis acicularis and Marsilea quadrifolia (for Eleocharition) were identified in the area (Table 3). Cluster analyses clearly separate this class and the Isoëto-Nanojuncetea class into the same cluster (Fig. 7).

Order Litorelletalia uniflorae Koch 1926 is the only one represented in Romania's vegetation, through a single alliance and a single plant community. Alliance Eleocharition acicularis Pietsch ex Dierßen 1975 is only represented by Eleocharidetum acicularis Koch 1926, a new phytocoenosis for the area, which occupies lentic, oligotrophic up to mesotrophic habitats. Eleocharidetum acicularis W. Koch 1926 was identified only on the Moldova Veche island, where it forms a narrow strip around ponds. It was only reported in August. The studied phytocoenoses are poor - consisting of only 14 species.

The phytogeographical spectrum reflects the good representation of cosmopolitan and circumpolar species, followed by Eurasian elements (Fig. 5). Only two alien species were 
reported: Paspalum paspalodes and Sagittaria trifoliata.
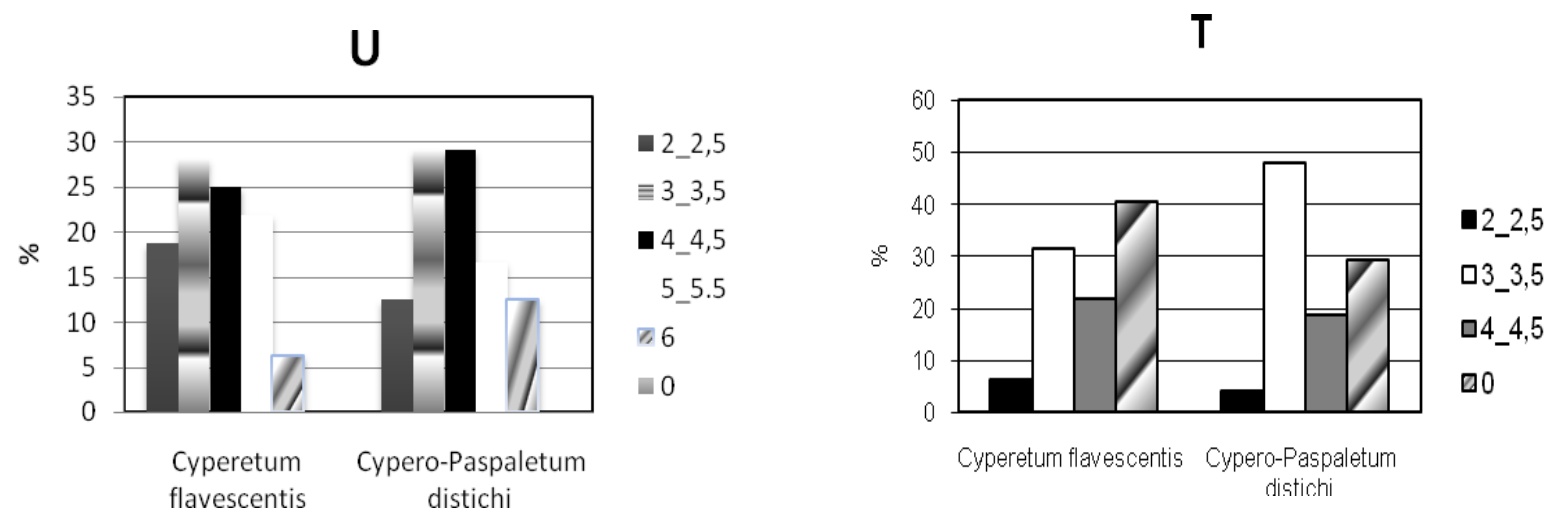

$\mathbf{R}$

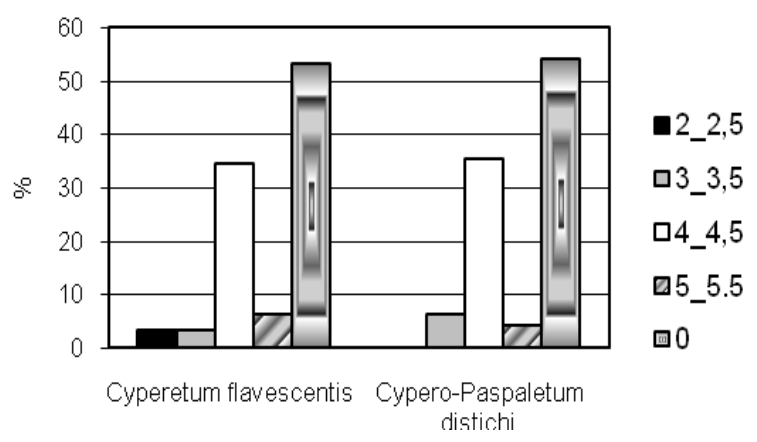

Fig. 4: The ecological spectrum of Isoëto-Nanojuncetea communities from the "Iron Gates" Natural Park regarding humidity $(U)$, temperature $(T)$, soil chemical reaction $(R)$

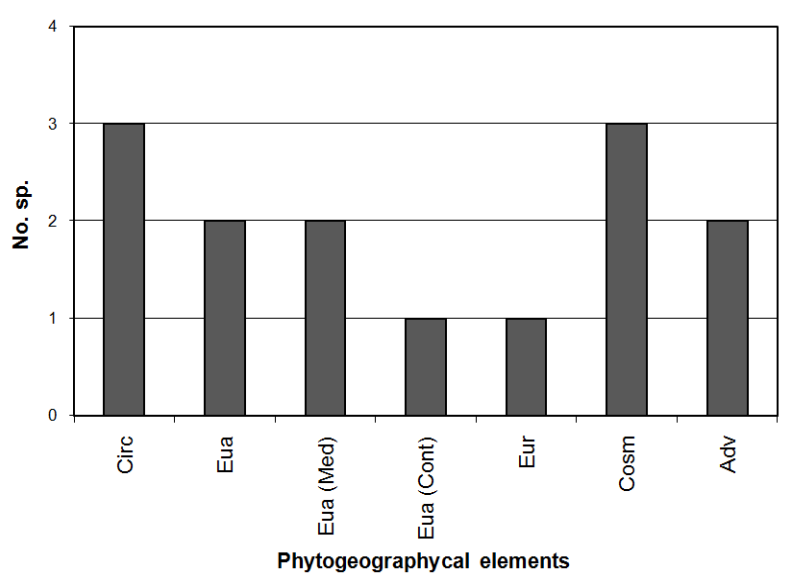

Fig. 5: The phytogeographical spectrum of the Eleocharidetum acicularis phytocoenosis from the "Iron Gates" Natural Park

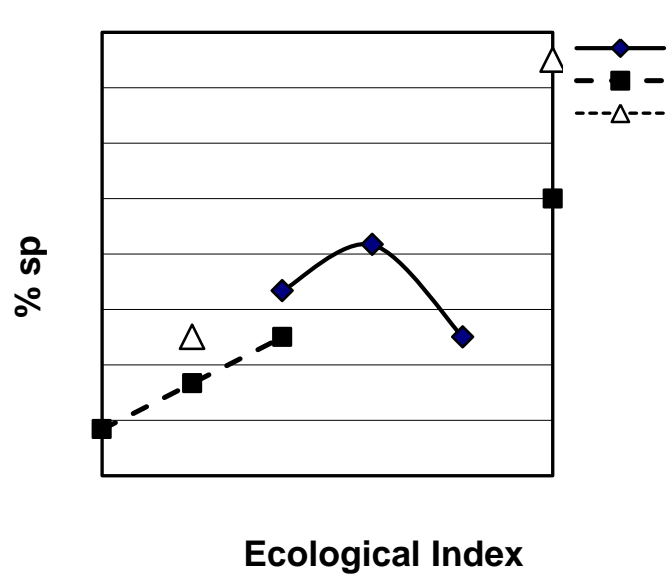

Fig. 6: The ecological spectrum of the Eleocharidetum acicularis phytocoenosis from the "Iron Gates" Natural Park 
Table 3: Plant communities of Cl. Isoëto-Nanojuncetea and Litorelletea from the "Iron Gates" Natural Park

\begin{tabular}{|c|c|c|c|c|c|c|c|c|c|c|c|c|c|c|}
\hline Relevé no. & 57 & 58 & 59 & 60 & 61 & 62 & 63 & 64 & 65 & 66 & 67 & 68 & 69 & 70 \\
\hline Relevé area $\left(\mathbf{m}^{2}\right)$ & 2 & 6 & 2 & 25 & 9 & 25 & 25 & 9 & 25 & 25 & 25 & 25 & 1 & 2 \\
\hline Total cover (\%) & 50 & 100 & 90 & 90 & 100 & 90 & 100 & 100 & 100 & 40 & 70 & $\mathbf{5 0}$ & 60 & 60 \\
\hline Cyperus flavescens & 3 & 5 & 4 & 4 & 3 & 2 & & 1 & . & & . & & + & + \\
\hline Paspalum paspaloides & + & . & . & 1 & . & 4 & 5 & 4 & 4 & 2 & 3 & 3 & 1 & + \\
\hline Cyperus glomeratus & . & . & . & $\cdot$ & . & $\cdot$ & . & . & . & . & . & . & . & . \\
\hline Cyperus serotinus & . & . & . & 1 & . & + & . & . & . & + & . & + & . & . \\
\hline Verbion supinae & & & & & & & & & & & & & & \\
\hline Verbena officinalis & . & & 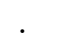 & & 1 & & & + & . & . & . & . & . & . \\
\hline Cyperus fuscus & . & & & + & & & & 1 & . & . & . & . & . & . \\
\hline Juncus bufonius & . & & & & 1 & & . & & . & . & . & . & . & . \\
\hline Mentha pulegium & . & 1 & + & . & 2 & + & . & + & + & . & . & . & . & . \\
\hline Tripleurospermum tenuifolium & & . & + & . & . & . & . & . & . & . & . & . & . & . \\
\hline Eleocharis acicularis & 1 & . & . & . & . & . & . & . & . & + & + & + & 3 & 4 \\
\hline Marsilea quadrifolia & . & . & . & . & . & . & . & . & . & 2 & 2 & 1 & + & . \\
\hline Amaranthus retroflexus & . & . & . & . & . & . & . & + & . & . & . & . & . & . \\
\hline Bidens cernua & . & . & . & . & + & . & + & + & 2 & . & . & . & . & . \\
\hline Bidens frondosa & . & . & . & . & . & + & + & & . & . & . & . & . & . \\
\hline Bidens tripartita & . & . & . & . & . & . & . & 1 & . & . & . & . & . & . \\
\hline Chenopodium album & . & . & . & . & . & . & . & + & . & . & . & . & . & . \\
\hline Chenopodium rubrum & . & . & . & . & . & . & . & + & . & . & . & . & . & . \\
\hline Echinochloa crus-galli & . & . & . & . & . & . & . & 1 & + & . & . & . & . & . \\
\hline Galega officinalis & . & . & . & . & . & . & . & . & . & . & . & . & . & . \\
\hline Polygonum lapathifolium & . & . & . & . & . & . & . & 1 & + & . & . & . & . & . \\
\hline Polygonum persicaria & . & . & . & . & . & + & + & . & . & . & . & . & . & . \\
\hline Ranunculus sceleratus & . & . & . & . & . & . & . & + & . & . & . & . & . & . \\
\hline Rorippa sylvestris & . & & . & & . & . & & + & . & & . & . & . & . \\
\hline Xanthium italicum & . & + & . & + & . & . & + & . & + & . & . & . & . & . \\
\hline Magnocaricion & & & & & & & & & & & & & & \\
\hline Galium palustre & . & . & . & . & . & . & . & + & . & . & . & . & . & . \\
\hline Poa palustris & . & + & . & . & + & . & . & . & . & . & . & . & . & . \\
\hline Bolboschoenion & & & & & & & & & & & & & & \\
\hline Schoenoplectus triqueter & 1 & . & . & . & . & . & . & . & . & . & . & . & 2 & + \\
\hline Alisma gramineum & . & • & . & & . & . & . & . & . & & . & . & . & + \\
\hline Alisma plantago-aquatica & . & . & . & + & . & . & . & . & . & + & + & + & . & . \\
\hline Butomus umbellatus & . & . & . & . & . & . & . & . & + & . & . & . & . & . \\
\hline Rumex hydrolapathum & . & . & . & . & . & . & . & + & . & . & . & . & . & . \\
\hline Berula erecta & . & . & . & . & . & . & . & + & . & . & . & . & . & . \\
\hline Lycopus europaeus & . & . & . & . & . & . & + & + & . & . & + & . & . & + \\
\hline Mentha aquatica & . & . & . & . & . & . & . & + & . & . & . & . & . & . \\
\hline Phragmites australis & . & . & . & . & . & . & . & . & 1 & . & . & . & . & . \\
\hline Typha laxmannii & . & . & . & + & . & . & . & . & . & . & . & 1 & . & . \\
\hline Agrostis stolonifera & . & . & . & 2 & . & & . & . & . & + & . & . & . & . \\
\hline Juncus articulatus & . & . & . & + & & + & . & . & . & . & . & . & . & . \\
\hline Juncus compressus & . & & . & & 2 & . & & & . & . & . & . & . & . \\
\hline Juncus inflexus & . & . & . & 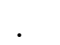 & + & . & & . & . & . & . & . & . & . \\
\hline Juncus tenuis & . & . & + & . & + & . & . & . & . & . & . & . & . & . \\
\hline Leontodon autumnalis & . & . & . & . & + & . & . & . & . & . & . & . & . & . \\
\hline Lotus corniculatus & . & + & . & . & . & . & . & . & . & . & . & . & . & . \\
\hline Lysimachia nummularia & & & & & & + & & . & . & & . & & . & . \\
\hline Lythrum salicaria & 1 & + & . & + & . & + & . & . & + & + & + & 1 & + & . \\
\hline Plantago lanceolata & . & + & & & + & & & & . & . & . & . & . & . \\
\hline Plantago major & 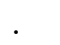 & & 1 & & + & & & + & . & . & . & . & . & . \\
\hline Potentilla anserina & . & & . & . & + & + & & . & . & . & . & . & . & . \\
\hline Potentilla reptans & . & . & . & . & . & + & . & . & . & . & . & . & . & . \\
\hline Pulicaria dysenterica & . & . & . & . & . & . & . & + & . & . & . & . & . & . \\
\hline Ranunculus repens & & & & & & & & + & . & . & . & . & . & . \\
\hline
\end{tabular}


Trifolium repens Ambrosia artemisiifolia Ammannia verticillata Amorpha fruticosa Bidens vulgata

Cyperus odoratus Erigeron strigosus Matricaria chamomilla Mentha verticillata Plantago media

Date and place of relevés: Ass. 19. Cyperetum flavescentis: rel. 57-58 - Moldova Veche island (31.07.2012), rel. 59, 61 - Liubcova (03.08.2012: 24.09.2012), rel. 60 - Moldova Veche island (23.09.2012); Ass. 20. Cypero-Paspaletum distichi: rel. 62 - Moldova Veche island (23.09.2012), rel. 63-65 - Pojejena (24.09.2012); Ass. 21. Eleocharidetum acicularis: rel. 66-70 - Moldova Veche island (23.09.2012).

From an ecological point of view, hygrophilous species $\left(\mathrm{U}_{6}\right)$ predominate. In relation to the temperature and chemical reaction $(\mathrm{pH})$ of the substrate, euriionic and eurithermal species are predominant. Among stenotopic species, moderately thermophilic $\left(\mathrm{T}_{4-4.5}\right)$ and micromesothermal ( $\left.\mathrm{T}_{3-3.5}\right)$, weakly neutrophilic-acidophilic $\left(\mathrm{R}_{4-4.5}\right)$ and acid-neutrophilic $\left(\mathrm{R}_{4-4.5}\right)$ species have a good representation (Fig. 6).

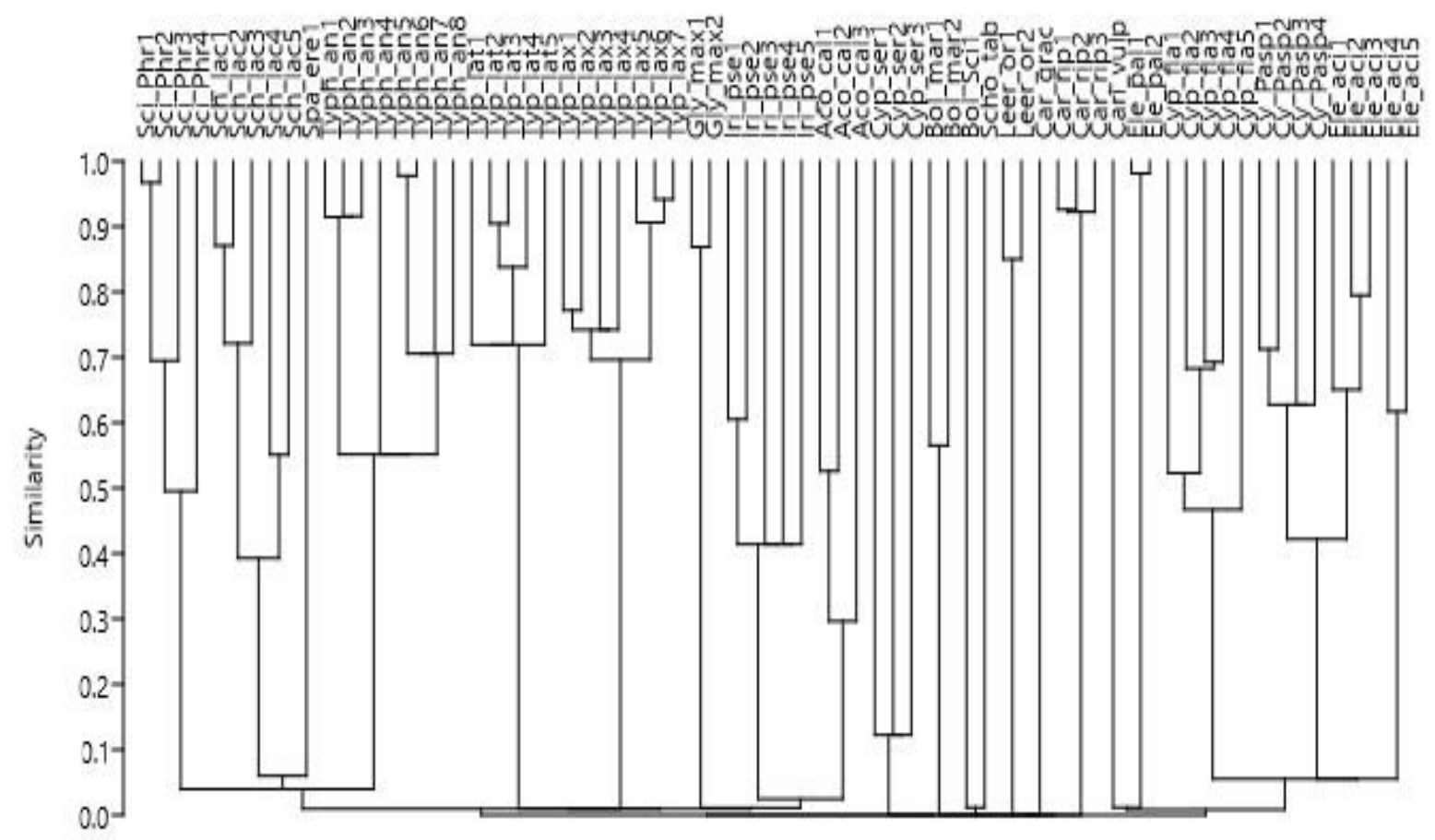

Fig. 7: Cluster analyses of swamp communities from the "Iron Gates" Natural Park

Habitats of European community interest represented by swamp vegetation in the "Iron Gates" Natural Park

Only one habitat of European community interest was identified in this study: $\mathbf{3 1 3 0}$ Oligotrophic to mesotrophic standing waters with vegetation of the Littorelletea uniflorae and/or Isoëto-Nanojuncetea CLAS. PAL.: $22.12 \times$ (22.31 and 22.32). It is represented by the plant communities: Cyperetum flavescentis W. Koch ex Aisinger 1933, Eleocharidetum acicularis W. Koch 1926, and Cypero-Paspaletum distichi Horvatic 1954.

The previously mentioned communities are present on the Moldova Veche island (all of 
them - about 2-3 hectares), in Pojejena and Liubcova (Cyperetum flavescentis only). They occupy narrow areas on the banks of the ponds. Important for this habitat is the presence of Marsilea quadrifolia (common water clover), a species of community importance[36, 37], also included in the IUCN Red List [43]. Some of the Red List species hosted by this habitat are: Schoenoplectus triqueter, Fimbristylis bisumbellata, Ammania verticillata and Cyperus serotinus [26].

\section{Discussions}

The database of the swamp vegetation of the "Iron Gates" Natural Park was enriched with 7 new associations and one subassociation. Related to previous records [12, 13, 29, 24, 21] 6 phytocoenotaxa assigned to these three classes, weren't retreived in our study. Matacă (2005) reported 44 associations throughout the Natural Park. By also taking into consideration a study on aquatic vegetation [20], which presented 23 aquatic plant associations (11 associations and one subassociation mentioned for the first time), the contribution of studies focusing on wetlands can be evidenced, which could be explained by the increased dynamics of this type of vegetation, but also by an easier access to the border area.

On the other hand, one of the associations, reported for the first time in Romania Cypero-Paspaletum distichi Horvatic 1954, is the consequence of the invasiveness of the Paspalum paspalodes, which was reported in the studied area as early as 2005 [1].

Regarding the ecological feature of these communities, they fit the profile of swamp formations, which is explained by the fluctuating water level, these phytocoenoses being flooded in the spring, while over time benefiting only from the soil moisture. This aspect is much more obvious for the associations of Isoëto-Nanojuncetea and Littorelletea uniflorae classes, which have a shorter vegetation period depending on soil moisture and temperature.

From the point of view of temperature preferences, species with Mediterranean characteristics are well represented; in fact, the influence of this climate in south-western Romania is well known [11].

The presence of the habitat 3130 Oligotrophic to mesotrophic standing waters with vegetation of the Littorelletea uniflorae and/or Isoëto-Nanojuncetea is surprising in a mountain area with a high tourism potential. In fact, this habitat is present only in more isolated areas, particularly on the Moldova Veche island. Horse grazing mainly affects the Marsilea quadrifolia species, as reported by Andrei et al. (2011) and Bodescu (2013).

According to the Red List elaborated by Oltean et al. (1994), 8 species are rare (Schoenoplectus triqueter, Cyperus serotinus, Fimbristylis bisumbellata, Juncus bulbosus, Sagittaria trifoliata, Trapa natans, Najas minor, Utricularia vulgaris), one species is vulnerable (Marsilea quadrifolia), and two species are near threatened (Salvinia natans and Ammannia verticillata). Sagittaria trifoliata is currently reported in Romania only in the Danube Delta: Sf. Gheorghe. According to the Red Book elaborated by Dihoru and Negrean (2009), one species is critically endangered (Juncus bulbosus), and two species are vulnerable (Azolla filiculoides, Veronica scardica).

Naturally, the phytocoenoses of associations belonging to the PhragmitoMagnocaricetea class host several species with conservation value, being much more widely distributed in the area. Only Fimbristylis bisumbellata and Ammannia verticillata are absent from the phytocoenoses of this class, being found in the Isoëto-Nanojuncetea class, along 
with Cyperus serotinus, Fimbristylis bisumbellat, Schoenoplectus triqueter. The Litorelletea class, only represented by the Eleocharitetum palustre association on very small surface areas - less than 0.2 ha, has a high conservation value because Marsilea quadrifolia is the best represented (AD 2) along with Cyperus serotinus and Schoenoplectus triqueter.

Among the 21 recorded alien plant species, Amorpha fruticosa, Paspalum distichum and Ambrosia artemisiifolia are the most dangerous invasive species in the surveyed area. It is possible that Amorpha fruticosa has already replaced representative areas of these swamp communities, as is the case in other wetlands [17].

\section{Conclusions}

The current study brings a substantial contribution to the knowledge of swamp vegetation in the "Iron Gates" Natural Park, enriching the plant inventory with 8 new coenotaxa for the area, one new association for the country, and reassessing the conservation value of plant communities as well as the impact of alien, mainly invasive species. Even if out of the 21 invasive species, only 3 currently pose serious problems, all potentially invasive species require close monitoring, and even more so as the greatest number of adventive species in relation to the total number of species were found in the Isoëto-Nanojuncetea class, which belongs to a habitat of community interest.

Acknowledgements: Financial support for this work was generously provided by the LIFE10 NAT/RO/740 project - Improving the conservation status of priority species and habitats in the "Iron Gates" wetlands.

\section{REFERENCES}

1. Anastasiu, P., Negrean, G., Milanovici, S., Cristurean, I., 2005, Plante adventive în Parcul Natural Porţile de Fier, Comunic. Simpozionul Şt., Grăd. Bot. "D. Brândză" şi Univ. din Bucureşti, Decembrie, 2010, rezumat.

2. Andrei, M., Roşca, V., Pascale, G., 2011, Starea actuală a Pădurii Letea, rezervație naturală de pădure virgină din Delta Dunării, Revista pădurilor, 126 (6): 18-21.

3. Bodescu, F., 2013, Raport final - Contract Nr. 11751/ 10.04.2013 Studiu privind amenințările asupra habitatelor de zone umede din zona transfrontalieră a Dunării în arealele Parcului Național Djerdap și Parcul Natural Porțile de Fier, "Managementul integrat al diversităţii biologice și de peisaj pentru dezvoltarea regională durabilă şi conectivitatea ecologică în Carpaţi”, http://www.bioregiocarpathians.eu/tl_files/bioregio/donwnloads_resources/Key\%20Outputs\%20and\%20Publication/WP6_6.2 _Wetlands\%20study_pilot\%20area_RO_PP07.pdf Cod CPV 79311200-9

4. Braun-Blanquet, J., 1964, Pflanzensoziologie, Springer Verlag, Berlin.

5. Călinescu, R., Coteț, P., Florea, N., Iancu, M., Martiniuc, C., Savu, A., Sârcu, I., Stoenescu, Şt., 1955, Geografia fizică a Republicii Populare Române, Bucureşti, Inst. Cerc. Geogr.

6. Cărăuş, I., 2012, Algae of Romania. A distributional checklist of actual algae. [Version 2.3], http://www.seaweedafrica.org/pdf/562DF96D13b4823893SWjO3A7893/50442.pdf.

7. Ciocârlan, V., 2000, Flora ilustrată a României. Pteridophyta et Spermatophyta, București, Edit. Ceres.

8. Coldea, Gh., 1991, Prodrome des associations végétales des Carpates du sud-est (Carpathes Roumaines), Doc. Phytosoc, Camerino, 13: 317-539.

9. Coldea, Gh. (ed.), Sanda, V., Popescu, A., Ştefan, N., 1997, Les associations végétales de Roumanie, Tome 1, Les associations herbacées naturelles, Presses Universitaires de Cluj, Cluj-Napoca.

10. Coldea, G., (ed.) 2012, Les associations végétales de Roumanie. Tome 2: Les associations anthropogénes, Presa Universitară Clujeană, Cluj-Napoca. 
11. Cristea, V., Gafta, D., 2015, The Mediterranean floristic element in the flora and vegetation of Romania. Fl. Medit., 25 (Special Issue): 65-76 doi: 10.7320/FlMedit25SI.065.

12. Csürös, S., Pop, I., Hodişan, I., Csűrös-Kaptalan, M., 1968, Cercetări floristice şi de vegetaţie între Orşova şi Eşelniţa, Contrib. Bot., VIII: 277-312.

13. Dihoru, G., Cristurean, I., Andrei, M., 1973, Vegetaţia dintre Valea Mraconiei-Depresiunea Dubova din Defileul Dunării, Acta Bot. Horti Bucurest. /1972-1973/: 353-423.

14. Dihoru, Gh., Negrean, G., 2009, Cartea roşie a plantelor vasculare din România, Bucureşti, Edit. Acad. Române.

15. Doniţă, N., Popescu, A., Paucă-Comănescu, M., Mihăilescu, S., Biriş, I. A., 2005, Habitatele din România, Bucureşti, Edit. Tehnică Silvică.

16. Doniţă, N., Popescu, A., Paucă-Comănescu, M., Mihăilescu, S., Biriş, I. A., 2006, Habitatele din România. Modificări conform amendamentelor propuse de România şi Bulgaria la Directiva Habitate (92/43/EEC), Bucureşti, Edit. Tehnică Silvică.

17. Doroftei, M., Anastasiu, P., 2014, Potential Impacts of Climate Change on Habitats and Their Effects on Invasive Plant Species in Danube Delta Biosphere Reserve, Romania. In: Rannow, S., Neubert, M. (eds) Managing Protected Areas in Central and Eastern Europe Under Climate Change. Advances in Global Change Research, vol 58, Springer, Dordrecht

18. Gafta, D., Mountford, J. O. (eds.), 2008, Manual de interpretare a habitatelor Natura 2000 din România/Handbook for interpretation of Natura 2000 habitats from România, Cluj-Napoca, Edit. Risoprint.

19. Glăvan, V., Geanana, M., 1972, Solurile. In: Atlasul complex „,Porţile de Fier”, Edit. Acad. Române.

20. Goia, I., Oprea, A. 2014, Particularities of the aquatic vegetation from "Porţile de Fier" Natura 2000 site, Transylv. Rev. Syst. Ecol. Res., 16: 93-122.

21. Grigore, S., Coste, I., 1978, Cercetări asupra vegetaţiei dintre Moldova Veche şi Pescari (judeţul CaraşSeverin), Banatica, Caiete Şti. Nat., Stud. Cerc.Geol., Geogr., Biol., Reşiţa, 7: 173-189.

22. Hammer, Ø., Harper, D.A.T., Ryan, P.D., 2001, Past: paleontological statistics software package for education and data analysis.

Palaeontologia Electronica 4: 9.http://palaeo-electronica.org/2001_1/past/issue1_01.htm

23. Matacă, S.Ş., 2005, Parcul Natural Porţile de Fier. Floră, vegetaţie şi protecţia naturii, Craiova, Edit. Universitaria.

24. Morariu, I., Danciu, M., Ularu, P., 1973, Die Vegetation der Flussinsel Moldova Veche, Acta Bot. Horti Bucurest. /1972-1973/: 465-499.

25. Mucina, L., Bültmann, H., Dierßen, K., Theurillat, J.-P., Raus, T., Čarni, A., Šumberová, K., Willner, W., Dengler, J., Gavilán García, R., Chytrý, M., Hájek, M., Di Pietro, R., Iakushenko, D., Pallas, J., Daniëls, F. J. A., Bergmeier, E., Santos., Guerra, A., Ermakov, N., Valachovič, M., Schaminée, J. H. J., Lysenko, T., Didukh, Ya. P., Pignatti, S., Rodwell, J. S., Capelo, J., Weber, H. E., Solomeshch, A., Dimopoulos, P., Aguiar, C., Freitag, H., Hennekens, S. M., Tichý, L., 2016, Vegetation of Europe: Hierarchical floristic classification system of vascular plant, bryophyte, lichen, and algal communities, Applied Vegetation Science, 19 (1): 3-264

26. Oltean, M., Negrean, G., Popescu, A., Roman, N., Dihoru, G., Sanda, V., Mihăilescu, S., 1994, Lista roşie a plantelor superioare din România, Stud., Sint., Doc. Ecol., Acad. Română-Inst. de Biologie, 1: 1-52.

27. Popescu, A., Ştefureac, Tr., 1976, Vegetationsforschungen aus dem Sektor Şviniţa-Trikule Eisernes tor Rumäniens, Acta Bot. Horti Bucurest. /1975-1976/: 341-368.

28. Popescu, A., Sanda, V., 1998, Conspectul florei cormofitelor spontane din România, Acta Bot. Horti Bucurest.: 1-336.

29. Raclaru, P., Alexan, M., 1973, Asociaţii vegetale palustre din Defileul Dunării, Baziaş-Pojejena, Stud. Cerc. Biol., Ser. Bot., 25 (2): 131-139.

30. Sanda, V., Şerbănescu, Gh., Zăvoianu, I., 1968, Aspecte ale florei şi vegetaţiei palustre din Clisura Cazanelor, Stud. Cerc. Biol., Ser. Bot., 20 (3): 217-224.

31. Sanda, V., Şerbănescu, G., Raclaru, P., Alexan, M., 1970, Contribuţii la cunoaşterea staţiunilor cu Acorus calamus L. şi însoţitoarele acesteia din România, Stud. Cerc. Biol., Ser. Bot., 22 (6): 481-489.

32. Sârbu, I., Ştefan, N., Oprea, A., 2013, Plante vasculare din România. Determinator ilustrat de teren (Vascular plants of Romania. An illustrated field guide), Publishing House Victor B Victor, Bucharest. 
33. Şerbănescu, G., Sanda, V., 1970, Cercetări asupra vegetaţiei de luncă şi dealuri între Cazanele Mari şi Plavişeviţa, Stud. Cerc. Biol., Ser. Bot., 22 (3): 171-178.

34. Todor, I., Gergely, I., Bârcă, C., 1971, Contribuţii la cunoaşterea florei şi vegetaţiei din zona Defileului Dunării între oraşul Moldova Veche şi comuna Pojejena (Judeţul Caraş-Severin), Contrib. Bot., XI: 203256.

35. Tutin, T.G., Heywwood, V.H., Burges, N.A., Moore, D.M., Valentine, D.H., Walters, S. M., Webb, D.A., 1964-1980, Flora Europaea, Cambridge, Cambridge Univ. Press, Vol. 1-5.

36. ***Council Directive 92/43/EEC of 21 May 1992 on the conservation of natural habitats and of wild fauna and flora (OJ L 206, 22.7.1992, p. 7), annex IIb.

37. ***Council Directive 92/43/EEC of 21 May 1992 on the Conservation of Natural Habitats and of Wild Fauna and Flora, [1992] OJ L 206, full text available at the European Commission's nature conservation homepage, http://europa.eu.int/comm/environment/nature/home.htm.

38. ***Harta geologică a României, Scara 1 : 200.000, Bucureşti: Inst. Geol. al României.

39. ***2013, Manuel d'interprétation des habitats de l'Union Européenne, EUR 28, European Commission, DG Environment, Nature, ENV B.3.

40. ***2011, Ordinul Ministrului Mediului şi Pădurilor nr. 2387 (M.Of. nr. 846 bis/29.11.2011) pentru modificarea Ordinului Ministrului Mediului şi Dezvoltării durabile nr. 1.964/2007 privind instituirea regimului de arie naturală protejată a siturilor de importanţă comunitară, ca parte integrantă a reţelei ecologice europene Natura 2000 în România: 3-100.

41. ***2013, Planul de management al Parcului Natural Portile de Fier. Ministerul Mediului, Apelor şi Pădurilor.

42. ***http://ww2.bgbm.org/europlusmed/ accessed on May $14^{\text {th }}, 2015$.

43. ***http://www.iucnredlist.org/details/161864/0

\section{PARTICULARITĂŢILE VEGETAŢIEI PALUSTRE DIN SITUL NATURA 2000 „PORȚILE DE FIER”, ROMÂNIA}

\section{(Rezumat)}

Lucrarea prezintă rezultatele investigaţiilor efectuate asupra vegetaţiei palustre de-a lungul malului românesc al Dunării, în zona sitului Natura 2000 Porţile de Fier (jud. Mehedinţi şi Caraş-Severin). Au fost identificate 20 de asociaţii vegetale si o subasociaţie, aparţinând claselor Phragmito-Magnocaricetea, IsoëtoNanojuncetea și Littorelletea. Studiile au condus la identificarea a 8 fitocenotaxoni noi pentru aria protejată, unul nou pentru țară - Cypero-Paspaletum distichi. Toate asociaţiile prezentate în lucrare sunt însoţite de tabele fitosociologice, analize cenotaxonomice, fitogeografice şi ecologice, în scopul evaluării stării de conservare a acestora, ca principal instrument în luarea viitoarelor decizii de management. 